\title{
EFECTO DEL CONSUMO DE HARINA DE MAÍZ FORTIFICADA CON ACIDO FÓLICO SOBRE LOS NIVELES DE FOLATOS SANGUÍNEOS EN MUJERES DE EDAD FÉRTIL
}

\author{
EFFECT OF FORTIFIED FLOUR CONSUMPTION \\ ON BLOOD FOLATE LEVELS IN WOMEN \\ OF CHILDBEARING AGE
}

\begin{abstract}
Maria Alejandra Sánchez P. (1), María del Carmen Esmer S. (1), Laura Martínez de V. (1), Nadia Varela G. (1), Ramón Valdez L. (2), Rosario Torres S. (1), Rocío López O. (2), Jesús Villarreal P. (3)

(1) Departamento de Genética.

(2) Departamento de Bioquímica.

(3) Servicio de Endocrinología Facultad de Medicina y Hospital Universitario "Dr. José Eleuterio González" Universidad Autónoma de Nuevo León. Monterrey, Nuevo León México.
\end{abstract}

\begin{abstract}
Fortification of foods is now widely used to prevent folate deficiency. Folic acid has been added to corn flour and its efficacy in the prevention of NTD has been proved. The aim of this study was to compare the effect of the intake of fortified corn-flour versus non fortified flour and to folic acid supplement, on blood folate levels in women of childbearing age. Forty-five women were randomized into three groups according to: (A) use of fortified flour $(\mathrm{n}=18)$, (B) non-fortified flour ( $\mathrm{n=17})$; (C) supplementation with $5 \mathrm{mg}$ of oral folic acid $(\mathrm{n}=10)$. All females answer a dietary and anthropometric questionnaire applied once a month during three months. Blood red cell (IE) and plasmatic (P)folate were measured at the beginning and at the end of the study; also the DNA analysis for the 677T mutation was performed. The corn flour was provided to groups $A$ and $B$ during three months, to be consumed as eight tortillas daily. Group C received $5.0 \mathrm{mg}$ tablet of folic acid (Valdecasas ${ }^{\circledR}$ ), once per week, during three months. IE and $P$ folate and hematocrito were significantly increased in women from group $C(p<0.05)$. Only plasmatic folic acid levels were significantly increased in women from groups $A$ and $B$. Genotype distribution was: 15.6\% homozygous TT, 42.2\% C/C and 42.2\% CT. Weekly administration of $5.0 \mathrm{mg}$ offolic acid is an effective way to increase blood folate levels and shows to be more efficient than fortified corn flour.
\end{abstract}

Key words: Fortified food; folic acid; neural tube defects; folate; MTHFR.

Este trabajo fue recibido el 8 de Junio de 2010 y aceptado para ser publicado el 15 de Mayo de 2011.

\section{INTRODUCCIÓN}

En la literatura médica existe evidencia indiscutible que el consumo de ácido fólico en el período periconcepcional previene en 50 y $75 \%$ defectos del tubo neural (DTN) por lo que se recomienda aumentar el consumo de alimentos ricos en folatos y/o fortificados (1). El Centro para el Control de las Enfermedades (CDC) de los Estados Unidos con la finalidad de aumentar los valores de folatos en la población, emitió la recomendación que todas las mujeres en edad fértil, sin antecedentes familiares de DTN deben consumir $400 \mu \mathrm{g}(0.4 \mathrm{mg}) \mathrm{de}$ ácido fólico por día para prevenir la ocurrencia y de 4.0 mg por día para prevenir la recurrencia en las mujeres con historia familiar positiva (2).

A pesar de que la deficiencia de folatos es más común en los países con economías menos desarrolladas, puede encontrarse en todos los niveles socioeconómicos de la población mundial. Las encuestas dietéticas sobre el patrón de alimentación de los individuos muestran que la ingesta de alimentos varía día con día, modificándose el consumo de los diversos nutrientes (2).

Estudios muestran una reducción del 25-30\% en 
la frecuencia de DTN donde aproximadamente la mitad es adjudicado al consumo de folatos. La relación folatos-DTN representa el único caso en el cual una malformación congénita puede ser prevenida simple y coherentemente (3).

Los niveles de ácido fólico están determinados tanto por su consumo en la dieta como por el nivel de funcionamiento de la enzima 5-10 metilenetetrahidrofolato reductasa (MTHFR). Se ha descrito que las mujeres homocigotas para la mutación C677T tienen mayor riesgo de descendencia con DTN (4).

México muestra una incidencia de estos defectos de 3 por 1000 nacimientos y ocupa el segundo lugar en frecuencia a nivel mundial, lo cual puede estar favorecido por características propias de la población tanto genéticas como en el hábito de consumo del ácido fólico. Las políticas nacionales actuales en desarrollo y salud promueven la alimentación y nutrición adecuada mediante la fortificación de alimentos, suplementación con vitaminas y diversificación de la dieta (5).

El consumo de esta vitamina debe formar un hábito en las mujeres pero los estudios realizados por Daly y cols confirman la falta de adhesión al consumo diario de la vitamina lo cual en la mayoría de los casos fue menor al 30\% (6). En un estudio previo, demostramos que la administración semanal de $5.0 \mathrm{mg}$ de ácido fólico por vía oral incrementaban significativamente los valores sanguíneos de ácido fólico en mujeres en edad fértil (7).

La fortificación de harinas y cereales se ha realizado en distintos países y su efectividad en elevar los niveles de ácido fólico y en prevenir la ocurrencia de DTN se ha demostrado en diversos estudios. En el 2001 un estudio en Estados Unidos mostró un 19\% de reducción en la prevalencia al nacimiento de defectos de tubo neural (8).

En México, a partir del 2000 se han fortificado los cereales y la harina de trigo y maíz de acuerdo a porcentajes de ingesta diaria recomendada (9). Se debe reconocer que en los hábitos de alimentación de nuestra población no se encuentra la ingesta diaria de cereal de hojuelas de maíz y si bien la harina de maíz en forma de tortilla constituye parte de nuestras costumbres alimentarias, con la fortificación actual se requerirían de 25 a 30 tortillas, para cumplir con las recomendaciones diarias de 400 mg (10) sin contar que el ácido fólico es termolábil.

El presente estudio se planteó por la necesidad de desarrollar estrategias para incrementar los valores sanguíneos de ácido fólico en nuestra población, de establecer el papel que el consumo harina de maíz fortificada pudiera tener como parte de estas medidas y compararlo con la administración de un suplemento oral en forma semanal para probar una forma de administración costo-eficiente.

\section{SUJETOS Y MÉTODOS}

Se diseñó un estudio prospectivo, longitudinal, experimental, aleatorizado y abierto. Con invitación abierta a mujeres de 12 a 45 años de edad que hubieran presentado la menarquia y sin antecedentes de productos malformados. Las participantes se eligieron por conveniencia entre las residentes de 3 poblaciones aledañas pequeñas con nivel socioeconómico bajo clasificado según los parámetros CONAPO-INEGI del área rural en el municipio de Dr. Arroyo del estado de Nuevo León, México, la cual fue seleccionada debido a que estudios previos habían demostrado una alta incidencia de DTN (11). Este proyecto fue aprobado por el Comité de Etica de la Facultad de Medicina de la Universidad Autónoma de Nueva León.

Fueron 45 mujeres que aceptaron participar previo consentimiento informado, aleatorizadas en tres grupos: al grupo A $(n=18)$ se les proporcionó harina de maíz vitaminada con $0.05 \mathrm{mg}$ de ácido fólico por cada 100 gramos de harina de maíz y se les adiestró en su preparación y proceso de cocción; al grupo $B(n=17)$ se le proporcionó harina de maíz no vitaminada y al grupo $\mathrm{C}(\mathrm{n}=10)$ se les indicó suplemento oral de ácido fólico (5.0 $\mathrm{mg}$ por semana). El estudio tuvo una duración de 3 meses, en este tiempo se les recomendó la ingesta de 8 tortillas/día (128 mg de ácido fólico) repartidas en 3 tiempos de comida de acuerdo a una dieta promedio de 2000 calorías; para la elaboración de las tortillas se utilizaron preparados comerciales de la misma marca de harina de maíz en formas vitaminada y no vitaminada, a las mujeres del grupo $\mathrm{C}$ se les proporcionó gratuitamente un frasco conteniendo tabletas de $5.0 \mathrm{mg}$ de ácido fólico para su consumo semanal (Valdecasas $\left.{ }^{\circledR}\right)$. Se les solicitó llevar registro diario de su ingesta, el cual se revisó mensualmente. También se aplicó una encuesta nutriológica validada, analizando la frecuencia alimentaria y recordatorio de 24 horas siendo aplicadas 3 encuestas en tres días diferentes en los tres grupos.

\section{Encuesta nutricional}

A todas las mujeres se les evaluó el estado nutricional previo al estudio y cada mes durante tres meses. Se registraron el peso y la talla con los cuales se calculó el Índice de Masa Corporal (IMC) de acuerdo con parámetros de la OMS. El análisis del contenido nutricional de la dieta se realizó por medio del paquete computacional Nutripac (2000) basado en referencias del Instituto Nacional de la Nutrición Salvador Zubirán, el cual permite desglosar todos los ingredientes que 
componen cada preparación y calcular el consumo individual de energía, hidratos de carbono, proteínas, grasa, ácido fólico, hierro, vitamina B 12, tiamina, riboflavina, niacina, calcio y zinc.

\section{Medición de folatos}

Se obtuvo una muestra de $5.0 \mathrm{ml}$ de sangre por punción venosa, previa limpieza del pliegue de flexión del codo con alcohol etílico. La muestra se colocó en 1 tubo con EDTA como anticoagulante al inicio y al final del estudio. Se determinó el hematocrito y después se tomó una alícuota de $0.1 \mathrm{ml}$ y se añadieron $2 \mathrm{ml} \mathrm{de}$ ácido ascórbico al $1 \%$ preparado inmediatamente antes de la dilución, para hemolizar las células sanguíneas y cuantificar el folato intraeritrocitario; durante todo el proceso las muestras fueron protegidas de la luz. Los 4.9 $\mathrm{ml}$ restantes fueron centrifugados a $1500 \mathrm{rpm}$ durante 10 minutos, obteniéndose el plasma para la medición de folato y vitamina B12. Las muestras fueron colocadas en tubos de plástico y mantenidos a $-35^{\circ} \mathrm{C}$.

Las determinaciones de folato y vitamina B12 se hicieron por radioinmunoensayo (RIA) de fase sólida (Dual Cont) obtenido de Diagnostic Products Corporation (DPC, los Angeles, California E. U. A.), estos incluyen un control liofilizado y controles CONG DPC bajo, medio y alto, para ambas vitaminas. Los valores de folato plasmático y de vitamina B12 fueron tomados directamente de la curva estándar. El valor intraeritrocitario se obtuvo multiplicando por el factor de dilución dividido entre el hematocrito. El valor de referencia del folato plasmático es de $0.028-0.038 \mathrm{pmol} / \mathrm{L}$, intraeritrocitario de 394.8-1579.2 nmol/L, y el valor de referencia de la vitamina B 12 es de 148-726 pmol/L.

\section{Análisis del polimorfismo del gen de la enzima MTHFR}

Se tomaron $5 \mathrm{ml}$ de sangre previo ayuno y se mantuvieron en refrigeración a $4^{\circ} \mathrm{C}$ hasta su determinación, la extracción de $\mathrm{ADN}$ se realizó mediante la técnica de TSNT. La mutación C677T consiste en una sustitución de alanina por valina en el codón 677 de la enzima MTHFR que puede ser reconocido por la enzima HinfI en el gen, por medio de la técnica de PCR-RFLP (reacción en cadena de la polimerasa y restricción de fragmentos de longitud polimórfica). La cantidad de ADN se cuantificó en geles de agarosa al $1 \%$ en el fotodocumentador Gel-Doc 2000. Para la reacción en cadena de la polimerasa (PCR) se utilizaron los iniciadores MTHFR-1 (5 -TGAAGGAGAAGGTGTCTGCGGA-3`) (3`-TGAAGGAGAAGGTGTCTGCGGA-5`) (12).

\section{Análisis estadístico}

Se compararon los grupos antes y después de la intervención, en la ingesta de nutrientes y los valores de

\section{FIGURA 1}

Estado nutricional de grupos de intervención antes y después de tres meses en el estado de Nuevo León

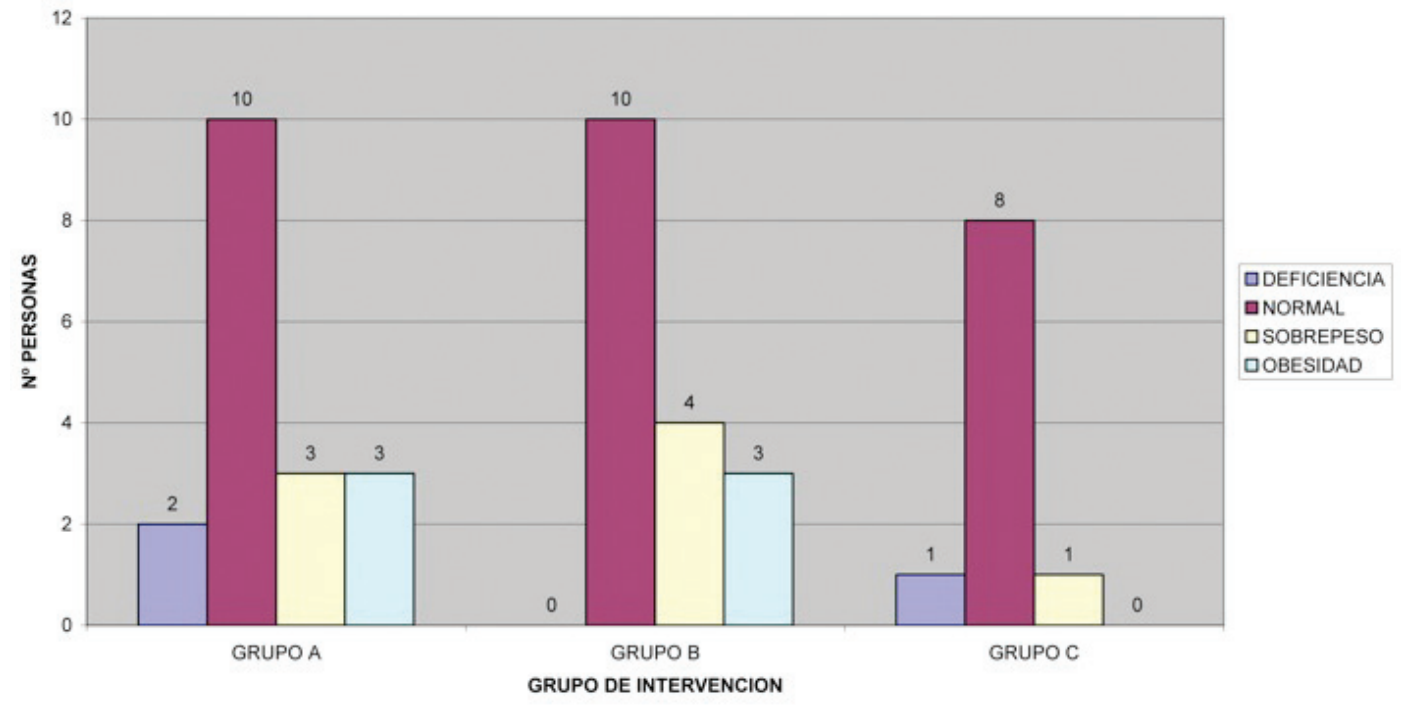


folato sanguíneo, utilizando la prueba de t de Student, Wilcoxon y análisis de varianza Duncan's para comparación múltiple en el programa estadístico "Statistics" y "NCSS and PASS trial 2001, Statistical Análisis and Grafics., UTA USA". El nivel de significancia fue ( $\mathrm{p}<0.05)$. Se calculó el porcentaje de mujeres que presentaban los diferentes genotipos para la MTHFR (homocigotos del alelo silvestre $\mathrm{CC}$, homocigotos del alelo polimórfico TT, heterocigotos CT) y se estimó la frecuencia alélica realizándose comparaciones mediante la prueba de Chi cuadrado.

\section{RESULTADOS}

Se realizó un diagnóstico nutricional al inicio y al final del estudio encontrando en el grupo A $55 \%$ eutróficos, $11 \%$ desnutridos, $17 \%$ con sobrepeso y $17 \%$ con obesidad. En el grupo B 59\% presentaron peso un normal, no se encontraron participantes con desnutrición pero en sobrepeso y obesidad se encontró un $24 \%$ y $17 \%$ respectivamente. En el grupo C el $80 \%$ mostraron un peso normal, $10 \%$ desnutridos y el $10 \%$ restante con sobrepeso. Las diferencias entre los grupos no fueron significativas (figura 1). Los grupos del estudio fueron similares en rango de edad (21-26 años).

En la encuesta dietarea de recordatorio de $24 \mathrm{hrs}$ al inicio y después del tercer mes se observó un incremento significativo en los grupos A y C para el consumo de hierro y tiamina $(\mathrm{p}<0.05$ y $\mathrm{p}<0.01$, respectivamente). En los grupos A y B se observó un aumento en la ingesta de hidratos de carbono $(\mathrm{p}<0.05)$ y folato $(\mathrm{p}<0.01$ y $\mathrm{p}<0.05)$ respectivamente. $\mathrm{El}$ análisis del contenido nutricional de la alimentación documentó que la mayoría del ácido fólico dietario proviene de los frijoles teniendo un promedio de consumo de $150 \mathrm{~g}$ al día. La población mostró un consumo diario mínimo de folatos de acuerdo a las recomendaciones diarias de folato dietético sugeridas para el género y edad (400 mg), mientras que las mediciones sanguíneas basales muestran valores normales en los diversos parámetros medidos.

El grupo A que usó harina vitaminada fue el único que incrementó significativamente el consumo de zinc y riboflavina los cuales estaban agregados en la harina, mientras que en el grupo $\mathrm{C}$ se observó un incremento significativo del consumo de calcio (tabla 1).

En los grupos A y B se incrementó significativamente la cianocobalamina y el FP $(\mathrm{p}<0.005)$. En las mujeres del grupo B se observó disminución del hematocrito lo cual refleja una disminución no significativa del consumo de hierro dietario (tabla 2). Los valores de folato (IE y P), el hematocrito y la cianocobalamina (B12) se incrementaron significativamente en el grupo C.

El análisis del polimorfismo C677T del gen de la

\section{TABLA 1}

\section{Consumo de macronutrientes, vitaminas y minerales al en los grupos de intervención en el estado de Nuevo León, México}

\begin{tabular}{|c|c|c|c|c|c|c|}
\hline & \multicolumn{2}{|c|}{ Harina vitaminada $(\mathrm{n}=18)$} & \multicolumn{2}{|c|}{ Harina no vitaminada $(n=17)$} & \multicolumn{2}{|c|}{ Suplemento $(n=10)$} \\
\hline & Cero meses & 3 meses & Cero meses & 3 meses & Cero meses & 3 meses \\
\hline Calorias (kcal) & $1481+705$ & $1699+487$ & $1255+393$ & $1507+458$ & $1016+273$ & $1273+550$ \\
\hline Carbohidratos (gr) & $238+101$ & $315+91 *$ & $221+80$ & $278+94 *$ & $151+39$ & $234+116$ \\
\hline Proteínas (gr) & $47+19$ & $57+19$ & $50+17$ & $57+19$ & $36+12$ & $47+16$ \\
\hline Grasa (gr) & $24+19$ & $24+9$ & $19+6$ & $18+7$ & $29+25$ & $15+6$ \\
\hline Acido Folico (ug) & $441.4+140.8$ & $664.1+234.1 * *$ & $542.6+222.1$ & $726.6+299.3^{*}$ & $346.6+169.4$ & $499.7+196.2$ \\
\hline Vitamina B12 (mg) & $1.06+0.59$ & $1.23+0.86$ & $1.23+1.75$ & $1.10+0.94$ & $0.82+0.97$ & $0.78+0.48$ \\
\hline Tiamina (mg) & $1.14+0.38$ & $1.77+0.47 * *$ & $1.34+0.43$ & $1.58+0.57$ & $0.91+0.27$ & $1.18+0.31^{*}$ \\
\hline Riboflavina (mg) & $0.69+0.68$ & $1.01+0.67^{*}$ & $0.60+0.27$ & $0.62+0.20$ & $0.50+0.17$ & $0.52+0.13$ \\
\hline Hierro (mg) & $2.92+4$ & $17.79+4$ & $15.45+5$ & $13.46+10$ & $9.52+2$ & $13.49+3$ \\
\hline Calcio (mg) & $487+161$ & $585+163$ & $641+346$ & $684+254$ & $332+121$ & $516+161^{* *}$ \\
\hline Zinc (mg) & $4.25+1.98$ & $18.30+9.82 * *$ & $4.73+1.82$ & $5.39+2.31$ & $3.87+2.47$ & $4.30+1.55$ \\
\hline
\end{tabular}


enzima MTHFR arrojó una frecuencia alélica (FA) de $37.8 \%$. La frecuencia en el total de mujeres homocigotas mutantes (TT) fue de $15.6 \%, 42.2 \%$ heterocigotas (CT) y $42.2 \%$ homocigotas normales (CC), lo cual se encontró en equilibrio según la ley de Hardy-Weinberg (X2 de 6.714, p=0.152). No existieron diferencias significativas entre los grupos al comparar la distribución de los genotipos.

En la tabla 3 se ilustran los valores promedio de los grupos con relación a las mediciones de folato plasmático e intraeritrocitario tomando en cuenta el genotipo, este análisis de realizó para determinar si la presencia de la mutación condicionaba una mejor respuesta a la intervención. Se observó que los individuos con el alelo C (normal) mostraron elevación del ácido fólico plasmático $(<0.05)$ mientras que los individuos con la mutación T elevan más bien los folatos intraeritrocitarios $(<0.05)$.

\section{DISCUSIÓN}

Hasta la fecha no se han realizado estudios sobre la utilidad y la aceptación de las harinas en especial la de maíz y su fortificación con ácido fólico que pudieran apoyar la prevención de enfermedades relacionadas con su deficiencia. El presente estudio muestra que en una población del área rural del noreste de México, el consumo de harina de maíz vitaminada (grupo A) eleva levemente los valores de folato intraeritrocitario, los cuales reflejan el estado crónico o de reserva tisular de folatos. Mientras que la administración semanal de 5 mg durante tres meses (grupo C) eleva no solo el valor plasmático e intraeritrocitario de folato, sino también el hematocrito en forma significativa lo cuál indica una respuesta de la médula ósea al aporte de la vitamina. La eficacia de esta medida también se evidenció en un estudio previo $(13,14)$ al observar una reducción en la incidencia de DTN (anencefalia 50\% y espina bífida $70 \%$ ) en el estado de Nuevo León.

Las mujeres que consumieron harina de maíz vitaminada y no vitaminada (grupos A y B) muestran un leve incremento no significativo en los valores de folato intraeritrocitario y plasmático. Es importante mencionar que en estos casos, el hematocrito disminuyó, por lo que podría tratarse de un $\square$ falso $\square$ incremento del folato intraeritrocitario, tal vez condicionado por deficiencia proteica ya que en estos lugares el consumo de proteínas de origen animal es bajo. La elevación de folato plasmático también podría haber sido condicionada por el consumo de polisacáridos no almidonados como los frijoles lo que se logró documentar en la encuesta nutricional y que en la región donde fue realizado el estudio es un alimento básico en la dieta. Houghton y colaboradores proponen que el incremento de la ingesta de polisacáridos no almidonados puede promover la síntesis intestinal de folatos, mostrando aumento del folato sérico (15). La

\section{TABLA 2}

Niveles sanguíneos por grupos de estudio al inicio y tras tres meses de intervención.

\begin{tabular}{|c|c|c|c|c|c|c|}
\hline & \multicolumn{2}{|c|}{ Harina vitaminada $(n=18)$} & \multicolumn{2}{|c|}{ Harina no vitaminada $(\mathrm{n}=17)$} & \multicolumn{2}{|c|}{ Suplemento oral $(n=10)$} \\
\hline & Basal & $\begin{array}{c}\text { Después de } \\
3 \text { meses }\end{array}$ & Basal & $\begin{array}{c}\text { Después de } \\
3 \text { meses }\end{array}$ & Basal & $\begin{array}{l}\text { Después } \\
3 \text { meses }\end{array}$ \\
\hline $\begin{array}{l}\text { Hematocrito } \\
\text { (normal: } 33-44 \% \text { ) }\end{array}$ & $37+6$ & $38+5$ & $39+5$ & $38+3$ & $36 .+5$ & $41+1 *$ \\
\hline $\begin{array}{l}\text { Acido fólico plasmático } \\
\text { (Referencia: } \\
0.028-0.038 \mathrm{pmol} / \mathrm{L})\end{array}$ & $0.017+0.004$ & $0.022+0.006^{*}$ & $0,016+0.004$ & $0,020+0.003 *$ & $0.016+0.005$ & $0.023+0.004 * *$ \\
\hline $\begin{array}{l}\text { A. fólico intraeritrocitario } \\
\text { (Referencia: } \\
394.8-1579.2 \mathrm{nmol} / \mathrm{L} \text { ) }\end{array}$ & $552.5+143.8$ & $577.8+132.9$ & $583.6+175.8$ & $639.6+141.8$ & $565.2+174.4$ & $629.8+88.0 * *$ \\
\hline $\begin{array}{l}\text { Vitamina B } 12 \\
\text { (Referencia: }\end{array}$ & & & & & & \\
\hline $148-726 \mathrm{pmol} / \mathrm{L})$ & $384+109$ & $644+190 * *$ & $361+102$ & $579+91 * *$ & $360+141$ & $667+160 * *$ \\
\hline $\begin{array}{l}* p>0.05 \\
* * p<0.005\end{array}$ & & & & & Fuente: D & ecta \\
\hline
\end{tabular}


ingesta de ácido fólico observada en esta población es probablemente una de las más altas en el mundo, por ejemplo Rodríguez-Castillo comentan que en zonas rurales de Costa Rica el consumo de frijol ha disminuido a 42.9g/día (16) mientras que en la población en México, particularmente en las áreas rurales es la base de la dieta, teniendo un consumo hasta de $150 \mathrm{~g} /$ día. Otras fuentes de folatos como el huevo, papas, chile y tomate también contribuyen a los niveles de consumo tan altos que se documentaron en las encuestas nutricionales.

Una ingesta elevada de ácido fólico no siempre se ve reflejada en los niveles plasmáticos e intraeritrocitarios, debido a diversos factores. El maíz pierde muchos de sus nutrientes desde el proceso de nixtamalización provocando pérdidas de vitaminas, en especial el ácido fólico, con un $46.3 \%$ de pérdida; a eso se debe de sumar el $20.7 \%$ que se pierde durante la nixtamalización. También disminuyen otras propiedades de la tortilla como el color, sabor y textura $(17,18)$. Los componentes vitamínicos que más se pierden en la transformación del maíz en tortilla son la riboflavina y el ácido fólico, cuyas pérdidas son elevadas especialmente durante la cocción.

Indudablemente que los nutrientes presentes en la harina vitaminada traerán un mejor estado nutricional a nuestra población, sin embargo, esta medida no es suficiente para la prevención de los DTN, también se debe de contemplar que existen comunidades que producen su propio maíz para autoconsumo y estos no serán beneficiados por las reformas de adición.

Sobre el estado nutricional no se encontró una diferencia significativa en la disminución o aumento de peso al inicio y al final del estudio entre las participantes, esto se debe a la intervención dietética dada durante el estudio, para el consumo de los diversos alimentos, sin incrementar la cantidad diaria del requerimiento de acuerdo a la edad y género.

Existen algunos estudios que indican una mayor respuesta al aporte de folatos en los individuos con genotipo TT. En nuestro estudio se observó que un mayor porcentaje de mujeres del grupo $\mathrm{C}$ eran homocigotos para el polimorfismo (TT), este grupo fue el que registró mayores cambios en términos de FP e IE de tal manera que los resultados obtenidos podrían estar modificados por esta diferencia en el genotipo.

En una publicación previa se estudió una muestra integrada por 250 mujeres mexicanas con una prevalencia del genotipo TT de $34.8 \%$ con una frecuencia alélica de 0.58; posteriormente, González y cols. reportaron en Yucatán, estado localizado al sur del

\section{TABLA 3}

Valores de folatos en mujeres en edad fértil comparados con el genotipo en Monterrey, Nuevo León antes y despues de la intervención

GENOTIPO

$\begin{array}{cc}\text { GRUPO A } & \text { GRUPO B } \\ \text { Vitaminada } & \text { Sin vitaminas } \\ \mathbf{N}=18 & \mathrm{~N}=\mathbf{1 7}\end{array}$

GRUPO A

\section{Basal Después de \\ 3 meses}

Basal

Después de

3 meses
GRUPO C

Suplemento

$\mathrm{N}=10$
Acido fólico plasmático

(Referencia: 0.028 - $0.038 \mathrm{pmol} / \mathrm{L}$ )

$\begin{array}{llllllc}\mathrm{C} / \mathrm{C} & 0.010 & 0.012 & 0.015 & 0.018 & 0.022 & 0.25 \\ \mathrm{C} / \mathrm{T} & 0.012 & 0.019 & 0.016 & 0.021 & 0.015 & 0.021 \\ \mathrm{~T} / \mathrm{T} & 0.011 & 0.014 & 0.014 & 0.019 & 0.012 & 0.020\end{array}$

A. fólico intraeritrocitario

(Referencia: 394.8 - $1579.2 \mathrm{nmol} / \mathrm{L}$ )

$\mathrm{C} / \mathrm{C}$
$\mathrm{C} / \mathrm{T}$
$\mathrm{T} / \mathrm{T}$

374.2

578.9

603.5

606.7

516.1

199.7

208.0

533.6

664.3

575.9

651.6

Fuente directa.
523.1

675.1
544.3 
país, una frecuencia del alelo mutado de 0.54 siendo la más elevada del país hasta ese momento; otro estudio, se encontró que la población mestiza de Chihuahua (localizada al norte de México) la frecuencia alélica fue de 0.38 y en la Tarahumara de la misma región de 0.39. La muestra poblacional analizada en el presente estudio (noreste de México) mostró una prevalencia del genotipo TT de $15.6 \%$ y la frecuencia del alelo mutado de 0.38. Estudios realizados en el estado de Nuevo León muestran una frecuencia mayor de homocigotos TT con una prevalencia de personas con genotipo TT del $20 \%$ (19). Probablemente la ubicación de los poblados al sur del estado en nuestro estudio pudiera explicar las diferencias encontradas en cuanto a prevalencia que de cualquier forma son significativamente mayores que en individuos estadounidenses, japoneses y africanos $(20,21)$.

En conclusión, el suplemento es la mejor estrategia para aumentar en forma constante los valores sanguíneos de folato en un grupo de mujeres del noreste de México, pero sin restar importancia a la fortificación de los alimentos (harina de maíz) sobre la nutrición de la población (22). La estrategia de administración semanal con dosis mayores de ácido fólico, además de elevar los valores sanguíneos, puede ser una alternativa para mejorar la adhesión al consumo de la vitamina y disminuir la incidencia de DTN.

\section{RESUMEN}

La fortificación de alimentos con ácido fólico puede ser efectiva en la prevención de los defectos del tubo neural, aunque no existen estudios que demuestren la eficacia de esta medida en la harina de maíz. El objetivo del estudio fue evaluar el efecto del consumo de harina de maíz vitaminada sobre los niveles de folatos sanguíneos, en comparación con la suplementación oral. Se incluyeron 45 mujeres en edad fértil aleatorizadas en tres grupos; grupo $\mathrm{A}(\mathrm{n}=18)$ harina vitaminada, grupo $\mathrm{B}$ $(\mathrm{n}=17)$ harina sin vitaminas, grupo $\mathrm{C}(\mathrm{n}=10)$ suplemento oral $5.0 \mathrm{mg}$ de ácido fólico una vez por semana, durante tres meses. A las participantes se les aplicó una encuesta nutricional y se les midió por radioinmunoensayo el folato intraeritrocitario (FIE) y plasmático (FP) al inicio y final del estudio y se determinó la presencia del polimorfismo C677T del gen MTHFR. En las mujeres con suplemento oral los niveles FIE y FP y el hematocrito se incrementaron significativamente $(\mathrm{p}<0.05)$, mientras que en los grupos A y B se registró un incremento significativo en los valores de FP pero no IE ni hematocrito. La ingesta de otras fuentes de ácido fólico se incrementó significativamente en los grupos A y B. El 15.6\% de las mujeres fueron homocigotas TT, $42.2 \%$ homocigotas CC y $42.2 \%$ heterocigotas CT. La administración de 5 mg de ácido fólico por semana es una medida más eficiente que la ingesta de harina de maíz vitaminada para elevar los niveles de folatos.

Palabras clave: Harina fortificada,; ácido fólico; defectos de tubo neural; folatos; MTHFR.

Dirigir la correspondencia a:

Dra.

Laura Martínez de Villarreal.

Departamento de Genética Facultad de Medicina.

Universidad Autónoma de Nuevo León.

Avenida Francisco I. Madero

Esquina Eduardo Aguirre Pequeño sin número

Colonia Mitras Centro.

Monterrey, Nuevo León México. C. P. 64460.

Teléfono/fax 0015283294217

E-mail: laelmar@yahoo.com.mx

Agradecimientos: Investigación realizada con recursos del departamento de Genética y Servicio de Endocrinología, Facultad de Medicina Universidad Autónoma de Nuevo León.

\section{BIBLIOGRAFÍA}

1. González VO. Defectos del tubo neural. Arch Neurocien Mex 2007;12(3):171-175

2. Firth Y, Murtaugh MA, Tangney C. Estimation of individual intakes of folate in women of childbearing age with and without simulation of folic acid fortification. J Am Diet Assoc 1998; 98: 985-8.

3. Pitkin RY Folate and neural tube defects. Am J Clin Nutr 2007; 85(suppl):285S-8S.

4. Van der Linder IJ, Afman LA, Heil SG, Blom HJ. Genetic variation in genes of folate metabolism and neural-tube defect risk. Proc Nutr Soc 2006;65(2):204-15.

5. Rosado JL. Camacho-Solís R, Bourges H. Adición de vitaminas y minerales a harinas de maíz y de trigo en México. Salud Publica Mex 1999;41:130-37

6. Daly S, Mills JL, Mohillo AM Minimum effective dose of folic acid for food fortification to prevent neural tube defects. Lancet 1997; 350:1666-9.

7. Martínez de Villarreal LE, Limón C, Valdés R, Sánchez A. Villarreal JZ. Efecto de la administración semanal de ácido fólico sobre los valores sanguíneos. Salud Publica Mex 2001;43(2):103-7.

8. Honein MA, Paulozzi LJ, Mathews TJ, Erickson JD, and Wong LY. Impact of folic acid fortification of the US food supply on the occurrence of neural tube defects. JAMA. 2001;285(23):2981-86.

9. Secretaría de Comercio y Fomento Industrial. 
Norma Oficial Mexicana (NOM-051-SCFI) Especificaciones Generales de Etiquetado para Alimentos y Bebidas no Alcohólicas preenvasados. 1994.

10. Casanueva E. La adición de nutrimentos a las harinas de maíz y de trigo. Cuadernos de nutrición 1999; 22:(2)69-76.

11. Instituto Naciopalde Estadística_Geografía e Informática INEGI www.inegi.gob.mx 2000.

12. Martínez de Villarreal L., Delgado Enciso I., Valdes Leal R.., Ortiz López R., Rojas Martínez A., Limón Benavides C., Sánchez Peña A., Ancer Rodríguez J, Barrera Saldaña H., Villarreal Pérez J. Folate Levels and MTHFR Genotype in mothers of offspring with neural tube defects a case-control study. Arch Med Res 2001;32:(4)277-82.

13. Martínez de Villarreal L., Arredondo Vázquez P., Hernández Herrera R., Velazco Campos R., Villarreal Pérez J. Decline of neural tube defects (NTD) cases alter a folic campaing in Nuevo León, México. Teratology 2002;66:(5):249-56.

14. Martínez de Villarreal L., Arredondo P., Hernández R, Villarreal Pérez J. Weekly administration of folic acid and epidemiology of neural tube defects. Matern Chile Health J 2006; 10:397-401.

15. Houghton LA., Green TJ. Donovan UM., Ginson RS., Stephen AM. Association Between dietary fiber intake and the folate status of a group of female adolescents. Am J Clin Nutr 1997; 66:1414-21.

16. Rodríguez Castillo L, Fernández Rojas X. Los frijoles (Phaseolus Vulgaris): Su aporte a la dieta del costarricense. Acta Med Costarric 2003; 45(3):120-5.

17. Arámbula Villa G, Barrón Ávila L, González
Hernández J, Moreno Martínez E, Luna Bárcenas G. Efecto del tiempo de cocimiento y reposo del grano de maíz (Zea Mayz L.) nixtamalizado, sobre las características físico químicas, geológicas, estructurales y texturales del grano, masa y tortilla de maíz. Arch Latinoam Nutr 2001; 51(2): 187-94.

18. Figueroa Cárdenas J, Acero Godínez MG, Lozano N, Guzmán Vasco A, Flores Acosta LM. y González Hernández J. Fortificación y Evaluación de tortillas de nixtamal. Arch. Latinoam Nutr 2001; 51(3):293302.

19. Aguirre Rodríguez A, Martínez de Villarreal LE, Velazco Campos MA, Sampallo Hernández E, Esmer Sánchez MC. Prevalencia en Nuevo León del Polimorfismo 677T del Gen MTHFR. Salud Publica Mex 2008; 50:(1):5-6.

20. Mills JL, Ramsbottom D, Molloy AM, Burke H, Weir DG. The thermolabile variant of methylentetrahydrofolate reductase and neural defect: An evaluation of genetic risk and the relative importance of the genotypes of the embryo and the mother. Am J Hum Genet 1999; 64:1045-55.

21. Mutchinick OM, López MA, Luna L, Waxman J, Babinsky VE. High prevalence of the thermolabile methylenetetrahydrofolate variant in México: a country with a very high prevalence of neural defects. Mol Genet Metab 1999; 68(4): 461-7.

22. Sánchez Peña MA., Varela Garza NS., Torres Sepúlveda MR., Valdés LeL r., Villarreal Pérez JZ., Martínez de Villarreal LE. Consumo de una harina de maíz fortificada y su Impacto en el Estado Nutricional de una población Infantil. Medicina Universitaria. 2005; 7:(29):2009-14. 